\title{
ILP Approaches to the Blockmodel Problem
}

Les Proll

School of Computing, University of Leeds, Leeds LS2 9JT, UK

email: lgp@comp.leeds.ac.uk 


\begin{abstract}
Blockmodelling is a method for identifying structural similarities or equivalences between elements which has applications in a variety of contexts, including multiattribute performance assessment. One criterion for forming blocks results in a difficult nonlinear integer programme. We give several integer linear programming formulations of this problem and provide comparative computational results. We show that methods of reducing symmetry proposed by Sherali and Smith are not effective in this case and propose an iterative approach in which the size of the problem is reduced.
\end{abstract}

Keywords: integer programming, nonlinear programming, blockmodel, symmetry

\title{
1 Introduction
}

The technique of blockmodelling originated in sociology [?] where it was used principally to identify structural similarities between elements in social networks [?, ?]. More recently it has been used to analyse multiattribute measures of performance in airlines [?], airports [?, ?, ?] and universities [?]. Blockmodelling provides an alternative to the strict ranking or 'league table' approach to relative performance assessment and, it is claimed [?], may have some advantages over the well established technique of data envelopment analysis [?]. In this context the performance, $p_{i}$, of unit $i$ is assessed via:

$$
p_{i}=\sum_{j} w_{j} v_{j}\left(x_{i j}\right)
$$

where $x_{i j}$ is the performance of unit $i$ on criterion $j, v_{j}($.$) are value func-$ tions mapping the performance measures to a common scale and $w_{j}$ are normalised, user-specified weights. Any given set of weights gives a ranking of the units. However there is likely to be some uncertainty as to what values should be assigned to the weights, leading to potential criticism of the ranking. There have been several approaches to analysing the sensitivity of the ranking to variations in the weights, including mathematical programming [?] and simulation [?]. The approach taken in block modelling is to recognise that the ranking may be sensitive and, instead, to look for sets of units whose performance scores are similar under different weights. To achieve this, probability distributions for the weights are proposed and distributions of the performance scores, $p_{i}$, obtained by simulation. From the latter, probabilistic estimates of the differences between the performance scores of pairs 
of units can be obtained. A pair of units can be viewed as having similar performance if the standardised difference between their scores is less than some threshold [?].

Essentially units in the scenario under study are represented by nodes in an undirected graph; an interaction between two units is represented by the presence of an edge between the corresponding nodes. Conventionally there is an edge connecting each node to itself. Two units are structurally similar if they have similar patterns of interaction. The blockmodel concept is to identify a partition of the graph into sets of mutually similar nodes, or blocks. Frequently there will be several possible partitions of the graph so that some means of choosing between them may be desirable. We might require that a block is a complete subgraph, i.e. that nodes in a block are structurally equivalent rather than structurally similar. However this may result in an undesirably large number of blocks. Jessop [?] proposes a criterion, maximum concentration, that favours the formation of a small number of large, dense blocks, i.e. blocks for which the intra-block edge density is close to 1. Justification of this approach and discussion of other approaches is given in [?]. Here we concentrate on the computational aspects of Jessop's approach.

\section{Jessop's Model and a Transformation}

The formulation of the block model problem suggested by Jessop [?] is:

$$
M A X H H I=\sum_{k=1}^{b}\left(\sum_{i=1}^{n} \lambda_{i k}\right)^{2}
$$

subject to

$$
\begin{gathered}
\sum_{k=1}^{b} \lambda_{i k}=1 \quad(i=1,2, \cdots, n) \\
\sum_{i=1}^{n} \sum_{j=1}^{n} x_{i j} \lambda_{i k} \lambda_{j k} \geq \beta\left(\sum_{i=1}^{n} \lambda_{i k}\right)^{2} \quad(k=1,2, \cdots, b) \\
\lambda_{i k}=0 \text { or } 1 \quad \forall i, k
\end{gathered}
$$

where $\lambda_{i k}=1$ if node $i$ is allocated to block $k, 0$ otherwise, $n$ is the number of nodes, $b$ is the maximum number of blocks to be formed, $x_{i j}$ is 1 if there is an edge connecting nodes $i$ and $j, 0$ otherwise, and $\beta$ is a parameter which represents the minimum acceptable block density. The value of $\beta$ is normally 
chosen to be close to 1 . In some circumstances there may be additional constraints on block sizes. For example, in sociological applications, it may be necessary to prohibit singleton blocks [?]; in design applications [?], maximum block size may be limited. Such constraints can be easily incorporated into the model.

Writing the number of nodes in block $k$ as $s_{k}=\sum_{i=1}^{n} \lambda_{i k}$, it can be seen that HHI is the sum of squares of block sizes. HHI represents a measure of concentration of the nodes into blocks and clearly favours the formation of a small number of large blocks. It is essentially the same as the HerfindahlHirschman Index [?], a popular measure of industrial concentration in an economy. When $\beta=1$, HHI is a multiple of the proportion of edges which are contained within blocks. It seems natural to prefer block structures for which this proportion is higher. Constraints (2) are set partitioning constraints which insist that every node is allocated to a single block. Constraints (3) may be rewritten:

$$
\frac{\sum_{i=1}^{n} \sum_{j=1}^{n} x_{i j} \lambda_{i k} \lambda_{j k}}{s_{k}^{2}} \geq \beta
$$

As $\sum_{i=1}^{n} \sum_{j=1}^{n} x_{i j} \lambda_{i k} \lambda_{j k}$ is the number of intra-block edges in block $k$, the left hand side of this constraint can be seen to represent the edge density of the block. Hence constraints (3) require the edge density of each block to reach a given threshold, $\beta$.

The principal difficulty in solving this model is that its continuous relaxation has a nonconvex feasible region. The direction of optimisation compounds the difficulty. Thus any attempt to solve this problem by branch and bound requires a global optimisation routine to handle the node subproblems, which potentially is computationally expensive. Commercial mathematical programming systems such as CPLEX, LINGO, MOSEK and XPRESS-MP [?] appear to support the solution of mixed integer quadratically constrained problems only in the convex case. However the binary nature of the variables allows the model to be linearized [?] by replacing the product term $\lambda_{i k} \lambda_{j k}$ by a binary variable $\omega_{i j k}$ together with the logical implications:

$$
\begin{aligned}
& \lambda_{i k}=0 \vee \lambda_{j k}=0 \Leftrightarrow \quad \omega_{i j k}=0 \\
& \omega_{i j k}=1 \quad \Leftrightarrow \quad \lambda_{i k}=1 \wedge \lambda_{j k}=1 .
\end{aligned}
$$

Taking account of the undirectedness of the graph edges, this gives:

$$
M A X H H I=\sum_{k=1}^{b}\left(\sum_{i=1}^{n} \lambda_{i k}+2 \sum_{i=1}^{n-1} \sum_{j=i+1}^{n} \omega_{i j k}\right)
$$


subject to

$$
\begin{gathered}
\sum_{k=1}^{b} \lambda_{i k}=1 \quad(i=1,2, \cdots, n) \\
2 \sum_{i=1}^{n-1} \sum_{j=i+1}^{n}\left(x_{i j}-\beta\right) \omega_{i j k} \geq(\beta-1) \sum_{i=1}^{n} \lambda_{i k} \quad(k=1,2, \cdots, b) \\
\omega_{i j k} \leq \lambda_{i k} \quad(i=1,2, \cdots, n-1 ; j=i+1, \cdots, n ; k=1,2, \cdots, b) \\
\omega_{i j k} \leq \lambda_{j k} \quad(i=1,2, \cdots, n-1 ; j=i+1, \cdots, n ; k=1,2, \cdots, b) \\
\omega_{i j k} \geq \lambda_{i k}+\lambda_{j k}-1 \quad(i=1,2, \cdots, n-1 ; j=i+1, \cdots, n ; k=1,2, \cdots, b) \\
\lambda_{i k}=0 \text { or } 1 \quad \forall i, k ; \quad \omega_{i j k} \geq 0 \quad \forall i, j, k
\end{gathered}
$$

which we denote Model 1. Note that the variables $\omega_{i j k}$ can be treated as continuous variables as constraints (8)-(10), together with the nonnegativity of $\omega_{i j k}$, force them to be binary in any feasible solution. The price paid for the linearization is a substantial increase in problem size. There are an additional $3 b n(n-1) / 2$ constraints and $b n(n-1) / 2$ variables as compared to the quadratic model (1)-(3). Whilst $n$ is fixed by the problem instance, choice of $b$ is a matter of judgement. We return to this point later.

\section{Symmetry Considerations}

The model detailed in Section 1 exhibits symmetry in that the labelling (1, $2, \cdots, b)$ of the blocks, whilst necessary for the specification of the model, is in reality arbitrary. Given a feasible allocation of nodes to blocks, any permutation of the block labels will also give a feasible solution. Symmetry is known to cause significant difficulties in tree search algorithms for the solution of discrete optimisation problems. Discussion and examples are given, for example, in Proll [?] and Sherali and Smith [?] in the case of integer linear programming, Proll and Smith [?] and Smith et al. [?] in the case of constraint programming, and Petrie et al. [?] in the case of hybrid constraint programming/linear programming.

Sherali and Smith suggest two approaches to reducing the effect of symmetry: model reformulation in terms of variables which eliminate the symmetry, and augmenting the model with constraints which impose a valid hierarchy on the (previously) feasible solutions. The first approach does not seem possible here without resorting to column generation approaches, as do Mehrotra and Trick [?], as the variables $\lambda_{i k}$ define which individual nodes 
are allocated to a particular block, which is what we need to know. Hence we employ the second approach.

An ordering of the blocks can be induced by insisting that they are indexed in nonincreasing order of size. This can be implemented by adding the constraints:

$$
\sum_{i=1}^{n} \lambda_{i 1} \geq \sum_{i=1}^{n} \lambda_{i 2} \geq \cdots \geq \sum_{i=1}^{n} \lambda_{i b}
$$

With this ordering, we can also impose:

$$
\sum_{i=1}^{n} \lambda_{i 1} \geq\lceil n / b\rceil
$$

We denote the augmented model Model 2.

In their work on the SONET problem, Sherali and Smith also suggested the use of:

$$
\sum_{i=1}^{n} i \lambda_{i 1} \geq \sum_{i=1}^{n} i \lambda_{i 2} \geq \cdots \geq \sum_{i=1}^{n} i \lambda_{i b}
$$

as a means of distinguishing between symmetric arrangements. In this case, suppose that, for example, we have an allocation in which nodes 1 and 2 are (fully) allocated to block $i$ and nodes 3 and 4 are allocated to block $j(j<i)$. For simplicity suppose that all other nodes are distributed across the remaining blocks, Then (12) allows this solution and also a solution in which nodes 3 and 4 are allocated to block $i$ and nodes 1 and 2 to block $j$, the distribution of other nodes remaining unchanged. Such solutions are not allowed by (14). As there are frequently many solutions containing blocks of the same size, there is some hope that (14) might be more effective than (12) in reducing the effects of symmetry. We denote the model comprising (1)-(11) and (14) as Model 3.

\section{Computational Experiments}

Computational experiments on Models 1, 2 and 3 were performed on seven problem instances ranging in size from 20 to 47 nodes using CPLEX 8.1 on a $2.8 \mathrm{GHz}$ processor running under Fedora Core 4 Linux. The parameter $\beta$ was set to 1.0 in all cases. Initial experiments suggested little hope of getting proven optimal solutions to these problems in a viable time. The smallest problem was stopped after 17 hours of cpu time using Model 1, at which 
time over 1 million nodes had been explored, the number of active nodes was still increasing at a substantial rate and the integrality gap was still $65 \%$. The same problem was allowed to run to proven optimality using Model 2; a process which took in excess of 131 hours. Clearly the branch and cut search has to be a truncated one. A maximum cpu time limit of 600 secs was set for problems 1-3 and 900 secs for problems 4-7.

Jessop [?] describes a constructive heuristic for the blockmodel problem in the case $\beta=1$. This was used to determine the maximum number of blocks, $b$, to be considered for each problem instance. Table 1 shows the problem data and the results obtained with the heuristic, where $V_{h}$ is the HHI value attained and $b_{h}$ is the corresponding number of blocks.

\begin{tabular}{|c|c|r|c|r|r|}
\hline Problem & Nodes & Edges & Density $(\%)$ & $V_{h}$ & $b_{h}$ \\
\hline 1 & 20 & 95 & 50 & 58 & 9 \\
2 & 30 & 117 & 27 & 104 & 12 \\
3 & 33 & 162 & 31 & 99 & 13 \\
4 & 40 & 282 & 36 & 254 & 9 \\
5 & 40 & 408 & 52 & 336 & 8 \\
6 & 47 & 393 & 36 & 321 & 10 \\
7 & 47 & 569 & 54 & 403 & 8 \\
\hline
\end{tabular}

Table 1: Problem data and heuristic solutions

Problem 1 arises from an analysis of the results of a particular season in the Barclays Premier League, the top division in English soccer [?]. Problem 2 arises from elective choices in an MBA programme [?]. Problem 3 is taken from a study of dwellings within a city [?]. Problems 6 and 7 arise from a multicriteria assessment of airport performance [?]; problems 4 and 5 are subsets of problems 6 and 7 respectively.

Table 2 shows that the potentially large MILP model is substantially reduced by CPLEX Presolve [?]. The reason for the magnitude of the reduction is clear. The insistence that the blocks are fully dense $(\beta=1)$ implies that if nodes $i$ and $j$ are not connected by an edge they cannot be in the same block. Hence the variables $\omega_{i j k}$ and associated constraints can be deleted, or indeed not generated. This will not be the case for other values of $\beta$.

A number of branching strategies were tried with no clear 'winner'. The results for Model 1, displayed in Table 3, were obtained with the CPLEX default strategies except that priority was given to branching in the up direction on the $\lambda$ variables and that the search was directed to concentrate on feasibility. 


\begin{tabular}{|c|r|r||r|r|}
\hline & \multicolumn{2}{|c||}{ Initial } & \multicolumn{2}{c|}{ After Presolve } \\
Problem & Rows & Columns & Rows & Columns \\
\hline 1 & 5168 & 1899 & 2871 & 1043 \\
2 & 15715 & 5992 & 5311 & 2164 \\
3 & 20651 & 7306 & 7235 & 2535 \\
4 & 21119 & 7389 & 9832 & 2898 \\
5 & 18777 & 6568 & 11505 & 3584 \\
6 & 29253 & 10161 & 13916 & 3960 \\
7 & 26008 & 9032 & 16103 & 4928 \\
\hline
\end{tabular}

Table 2: Problem size

\begin{tabular}{|c|r|r|r|r|r|r|r|}
\hline Problem & $V_{0}$ & $V_{1}$ & $\begin{array}{r}\text { Best } \\
\text { HHI }\end{array}$ & $N_{o}$ & $N_{e}$ & $N_{a}$ & $\begin{array}{r}\text { Upper } \\
\text { Bound }\end{array}$ \\
\hline 1 & 210.00 & 210.00 & 78 & 5904 & 20411 & 1974 & 152 \\
2 & 264.00 & 262.50 & 128 & 2843 & 3721 & 307 & 224 \\
3 & 357.00 & 355.92 & 117 & 0 & 1271 & 344 & 325 \\
4 & 604.00 & 602.55 & 276 & 1592 & 1619 & 341 & 543 \\
5 & 856.00 & 855.00 & 340 & 273 & 928 & 301 & 782 \\
6 & 833.00 & 833.00 & 337 & 390 & 461 & 154 & 798 \\
7 & 1185.00 & 1185.00 & 505 & 440 & 579 & 183 & 1111 \\
\hline
\end{tabular}

Table 3: Results for Model 1

In Table $3, V_{0}$ is the objective value of the LP relaxation, $V_{1}$ is the objective value after the addition of cuts, Best HHI is the objective value of the best integer feasible solution found, $N_{o}$ is the number of the node at which this solution was found, $N_{e}$ is the number of nodes explored prior to termination, $N_{a}$ is the number of active nodes remaining at termination.

The LP relaxation is clearly quite weak and not much improved by CPLEX cuts, despite there being of the order of 200 - 400 cuts generated at the root node. Nor are these cuts effective at later nodes. Progress in the search is characterized by a slow rate of reduction in the upper bound. The experiments suggest that it is relatively easy to find feasible solutions, a high proportion of which are found by CPLEX's heuristic.

Table 4 shows the results obtained with Models 2 and 3. Sherali and Smith showed that incorporating a similar set of symmetry breakers in their model for the SONET problem [?] leads to substantial improvement in performance. This is not the case here; neither Model 2 nor Model 3 perform as well as Model 1. However it may be noted that Sherali and Smith were able 
to run their instances of the SONET problem to proven optimality. This is not the case for the problem studied here as the search necessarily has to be truncated because of the observed slow progress towards proven optimality.

\begin{tabular}{|c|r|r|r||r|r|r|}
\hline & \multicolumn{3}{|c||}{ Model 2 } & \multicolumn{3}{|c|}{ Model 3 } \\
Problem & $V_{0}$ & $V_{1}$ & HHI & $V_{0}$ & $V_{1}$ & HHI \\
\hline 1 & 210.00 & 208.14 & 78 & 210.00 & 208.41 & 70 \\
2 & 264.00 & 260.40 & 110 & 264.00 & 261.45 & - \\
3 & 357.00 & 355.63 & 107 & 357.00 & 355.48 & 89 \\
4 & 604.00 & 601.40 & 238 & 604.00 & 601.81 & 216 \\
5 & 856.00 & 854.81 & 246 & 856.00 & 853.97 & 294 \\
6 & 833.00 & 831.12 & 283 & 833.00 & 831.45 & 321 \\
7 & 1185.00 & 1183.08 & 367 & 1185.00 & 1183.73 & 355 \\
\hline
\end{tabular}

\section{Table 4: Results for Models 2 and 3}

Sherali and Smith observed that CPLEX's heuristic finds it much more difficult to find feasible solutions when the symmetry breakers are present, particularly early on in the search. The experiments reported here echo this. A feasible solution of Model 1 was found at the root node for each problem and, in four of the seven cases, was better than that found by Jessop's heuristic. A first feasible solution of Models 2 and 3 was never found at the root node and, for the larger problems in particular, took a significant amount of the limited search time to find, for example Model 2 took 542 seconds to find a first IFS for problem 6. For each problem, the solution found was worse than that found by Jessop's heuristic. This goes some way to explaining why the use of the symmetry breakers does not appear beneficial in a truncated search. Trick [?] discusses other reasons why tightening the LP relaxation of an ILP at the modelling stage may not be beneficial.

It is easily shown [?] that, given a feasible solution, any improved feasible solution will have an HHI value which is better by at least 2. This fact, together with the HHI value found by the heuristic, can be used to set cutoffs which potentially prune the search tree. Given the comments on first feasible solutions above, this may be expected to be particularly advantageous to Models 2 and 3. However Table 5, which shows the objective value of the incumbent at termination for Models 1, 2 and 3 obtained using this cutoff strategy, does not support this expectation. The performance of Models 2 and 3 remains worse than that of Model 1, failing to improve on the initial solution in the majority of cases. Nor does the performance of Model 1 
consistently improve because of the cutoff.

\begin{tabular}{|c|c|c|c|c|c|c|c|}
\hline \multirow[b]{3}{*}{ Problem } & \multirow{3}{*}{$\begin{array}{l}\text { Initial } \\
\text { Cutoff }\end{array}$} & \multicolumn{2}{|c|}{ Model 1} & \multicolumn{2}{|c|}{ Model 2} & \multicolumn{2}{|c|}{ Model 3} \\
\hline & & Best & Upper & Best & Upper & Best & Upper \\
\hline & & HHI & Bound & HHI & Bound & HHI & Bound \\
\hline 1 & 58 & 78 & 153 & 78 & 148 & 74 & 156 \\
\hline 2 & 104 & 126 & 223 & 116 & 230 & 118 & 248 \\
\hline 3 & 99 & 119 & 325 & 99 & 333 & 99 & 344 \\
\hline 4 & 254 & 274 & 540 & 262 & 565 & 254 & 557 \\
\hline 5 & 336 & 352 & 854 & 336 & 790 & 336 & 789 \\
\hline 6 & 321 & 339 & 808 & 321 & 797 & 321 & 805 \\
\hline 7 & 403 & 503 & 1113 & 403 & 1135 & 403 & 1153 \\
\hline
\end{tabular}

Table 5: Results with Cutoffs Imposed

Thus our experience reinforces the warnings of Ragsdale and Shapiro [?] that use of such cutoffs may, in fact, degrade performance of a branch and cut search, particularly when the search is truncated.

Table 5 also shows that Models 2 and 3 are not especially successful in decreasing the upper bound on the objective function compared to Model 1. Table 6 shows the progress of Models 1 and 2 on problem 2 at approximately 600 second intervals. Progress on the other problems is similar and does not suggest that the symmetry breaking constraints become more beneficial when the search is less truncated.

\begin{tabular}{|r|r|c|c|c||c|c|c|c|}
\hline & \multicolumn{2}{|c|}{ Model 1 } & \multicolumn{2}{c||}{ Model 2 } & \multicolumn{2}{c|}{ Model 1 } & \multicolumn{2}{c|}{ Model 2 } \\
& \multicolumn{3}{|c|}{ no initial cutoff } & \multicolumn{3}{c|}{ with initial cutoff } \\
Time & Best & Upper & Best & Upper & Best & Upper & Best & Upper \\
(secs) & HHI & Bound & HHI & Bound & HHI & Bound & HHI & Bound \\
\hline 0 & 98 & 262 & - & 261 & 104 & 262 & 104 & 261 \\
600 & 128 & 224 & 110 & 225 & 126 & 223 & 116 & 230 \\
1200 & 128 & 219 & 114 & 219 & 126 & 216 & 116 & 217 \\
1800 & 136 & 215 & 122 & 215 & 126 & 212 & 128 & 214 \\
2400 & 136 & 211 & 128 & 212 & 126 & 209 & 128 & 210 \\
3000 & 136 & 208 & 128 & 208 & 128 & 206 & 128 & 207 \\
3600 & 136 & 206 & 132 & 207 & 128 & 204 & 128 & 205 \\
\hline
\end{tabular}

Table 6: Progress of Models 1 \& 2 on Problem 2

The preprocessing phase in CPLEX 8.1 includes an option, which does not appear to be available in other MILP solvers, to detect symmetry patterns 
and to add symmetry breaking cuts to an MILP model. By default this option is switched off, as was the case for the experiments described earlier. Table 7 displays results for Model 1 with this option switched on. The results show that the CPLEX symmetry cuts are effective in tightening the LP relaxation of Model 1, resulting in substantially decreased initial upper bounds in all but problem 6. Better values of the objective function are obtained for problems 2 - 5, including a proven optimal solution to problem 2 , but a worse value for problem 7 . The case of problem 6 is a strange one as CPLEX does not detect a symmetry pattern even though one obviously exists. There do not appear to be any features of problem 6 that clearly distinguish it from the other problems which might explain this behaviour. Note also that the presence of these symmetry breaking cuts does not affect the ability of CPLEX's heuristic to find an IFS at the root node in every case.

\begin{tabular}{|c|c|r||r|}
\hline Problem & $V_{1}$ & $\begin{array}{r}\text { Best } \\
\text { HHI }\end{array}$ & $\begin{array}{r}\text { Upper } \\
\text { Bound }\end{array}$ \\
\hline 1 & 136.00 & 78 & 94 \\
2 & 144.07 & 136 & 136 \\
3 & 198.10 & 125 & 162 \\
4 & 415.17 & 280 & 339 \\
5 & 667.35 & 398 & 553 \\
6 & 833.00 & 337 & 799 \\
7 & 857.47 & 481 & 767 \\
\hline
\end{tabular}

Table 7: Results for Model 1 with Symmetry Preprocessing

\section{Alternative Approaches}

As any solution of Model 1 is a partition of a fixed number of nodes into distinct blocks, the HHI value of a solution, which is a quadratic function of block size, will tend to increase as the number of blocks decreases. It is easily shown that an upper bound on HHI for a problem with $n$ nodes and exactly $b$ blocks is $(n-b+1)^{2}+b-1$, which is strictly increasing as $b$ decreases. However it is not true, in general, that the maximum value of HHI attainable by a solution in $b$ blocks is at least as large as the maximum value of HHI attainable by a $b+1$ block solution. Nevertheless it would seem reasonable to allow the tendency of HHI to increase with decreasing number of blocks to influence the search by giving preference to solutions with a smaller number 
of blocks. One approach is to introduce variables $\delta_{k}$ representing the presence $\left(\delta_{k}=1\right)$ or absence $\left(\delta_{k}=0\right)$ of block $k$ and to replace (5)-(11) by:

$$
M I N Z=Q\left(\sum_{k=1}^{b} \delta_{k}\right)-\sum_{k=1}^{b}\left(\sum_{i=1}^{n} \lambda_{i k}+2 \sum_{i=1}^{n-1} \sum_{j=i+1}^{n} \omega_{i j k}\right)
$$

subject to (6)-(11) and:

$$
\begin{gathered}
\sum_{i=1}^{n} \lambda_{i k} \leq\left(n-b_{L}+1\right) \delta_{k} \quad(k=1,2, \cdots, b) \\
\delta_{k}=1 \quad\left(k=1, \cdots, b_{L}\right) \\
\delta_{k}=0 \text { or } 1 \quad\left(k=b_{L}+1, \cdots, b\right)
\end{gathered}
$$

where $b_{L}$ is a lower bound on the number of blocks. We denote this Model 4. Unless the edge density of the graph is at least $\beta$, in which case the problem is trivial, $b_{L} \geq 2$. Setting $Q=n^{2}+1$ gives pre-emptive priority to minimising the number of blocks [?]. Results for Model 4 under similar conditions to those used in the experiments with Model 1 recorded in Table 3 are given in Table 8 . The only change to the conditions was that the branching strategy gave priority to the $\delta$ variables over the $\lambda$ variables and, within the $\delta$ variables, to the down direction, i.e. to eliminating a block.

\begin{tabular}{|c|r|r|r|r|}
\hline Problem & $V_{0}$ & $V_{1}$ & Blocks & Best HHI \\
\hline 1 & 1145.97 & 1795.40 & 6 & 78 \\
2 & 8136.02 & 8191.59 & 10 & 136 \\
3 & 7471.90 & 8378.33 & 10 & 125 \\
4 & 8160.87 & 12204.00 & 8 & 280 \\
5 & 5952.31 & 8027.63 & 6 & 390 \\
6 & 12909.30 & 19057.00 & 9 & 353 \\
7 & 8615.57 & 12075.00 & 6 & 519 \\
\hline
\end{tabular}

Table 8: Results for Model 4

For Model 4, the CPLEX default cut strategy is much more effective, both at the root node, as indicated in Table 8, and throughout the search. Model 4 finds the same solution as Model 1 for problem 1 but gives a better solution on termination for all the other problems.

Models 1-4 require the maximum number of blocks across which the nodes are to be allocated to be fixed. In the experiments previously reported in this paper, this was fixed at the number of blocks formed in the solution provided 
by Jessop's heuristic. The relatively good results provided by Model 4 suggest that looking for solutions with a small number of blocks is productive. Disaggregating (15) gives a two stage process, firstly minimising the number of blocks via Model 5 and then maximising HHI via Model 1. This has some advantage in that the search space for Model 1 is reduced by the elimination of $n(n-1) / 2$ constraints and $n+n(n-1) / 2$ variables for a reduction of 1 in $b$. Model 5 is:

$$
M I N \quad \sum_{k=1}^{b} \delta_{k}
$$

subject to (6)-(11), (15)-(17). It is run iteratively to allow the bounds $b_{L}, b$ on the number of blocks to be revised. The procedure is:

Set $b_{L}=2, b=b_{h}$, terminate $=$ false

While not terminate do

Solve Model 5

If Model 5

(i) proves no IFS exists, terminate $=$ true

(ii) finds an IFS with $b^{*}$ blocks

$$
\begin{aligned}
& b \leftarrow b^{*}-1 \\
& b_{L} \leftarrow\left\lceil V_{1}\right\rceil
\end{aligned}
$$

(iii) does not find an IFS, $b_{L} \leftarrow\left\lceil V_{1}\right\rceil$

If $b_{L}=b^{*}$ terminate $=$ true

Solve Model 1

Model 5 is run until the earliest of the following events occurs: (i) an integer feasible solution is found, (ii) 100 nodes of the search tree have been explored, (iii) 60 seconds cpu time have been used. The allowed run time of Model 1 is then fixed so that, for comparison purposes, the total cpu time is the same as that allowed in the experiments reported in Table 3. The sequence of results obtained with Model 5 are displayed in Table 9. 


\begin{tabular}{|c|r|r|c|r|r|r|}
\hline Problem & $b_{L}$ & $b_{U}$ & $V_{1}$ & $b^{*}$ & HHI & secs \\
\hline 1 & 2 & 8 & 3.20 & 8 & 56 & 0.25 \\
& 4 & 7 & 4.15 & 7 & 64 & 0.44 \\
& 5 & 6 & 5.00 & 6 & 68 & 0.28 \\
& 5 & 5 & - & - & - & 1.77 \\
\hline 2 & 2 & 11 & 9.28 & 11 & 94 & 1.61 \\
& 10 & 10 & 10.00 & 10 & 134 & 1.02 \\
\hline 3 & 2 & 12 & 7.14 & 12 & 97 & 6.77 \\
& 8 & 11 & 8.51 & 11 & 107 & 14.51 \\
& 9 & 10 & 9.00 & 10 & 121 & 2.46 \\
& 9 & 9 & 9.00 & - & - & 60.00 \\
\hline 4 & 2 & 8 & 6.03 & 8 & 242 & 15.95 \\
& 7 & 7 & - & - & - & 1.25 \\
\hline 5 & 2 & 7 & 4.13 & 7 & 256 & 4.85 \\
& 5 & 6 & 5.03 & 6 & 332 & 2.11 \\
\hline 6 & 2 & 9 & 6.15 & 9 & 293 & 19.78 \\
& 7 & 8 & - & - & - & 5.77 \\
\hline 7 & 2 & 7 & 4.30 & 7 & 339 & 11.80 \\
& 5 & 6 & 5.02 & 6 & 419 & 7.42 \\
\hline
\end{tabular}

Table 9: Sequential bound determination

In Table 9, a $V_{1}$ value of - denotes that the LP relaxation is infeasible, a $b^{*}$ value of - denotes that it is proven that no IFS exists within the bounds on the number of blocks. In most cases an IFS is found without branching. Table 10 gives the results obtained in the final run of Model 1 with the maximum number of blocks to be formed determined as above.

\begin{tabular}{|c|r|r|r|r|r|r|r|}
\hline \multirow{2}{*}{ Problem } & \multicolumn{2}{|c|}{ After Presolve } & & & & $\begin{array}{r}\text { Best } \\
\text { Rows }\end{array}$ & $\begin{array}{r}\text { Upper } \\
\text { Bound }\end{array}$ \\
\hline 1 & 1910 & 690 & 210.00 & 207.65 & 6 & 78 & 117 \\
2 & 4430 & 1470 & 264.00 & 257.83 & 10 & 136 & 224 \\
3 & 5573 & 1950 & 357.00 & 353.44 & 10 & 125 & 316 \\
4 & 8744 & 2576 & 604.00 & 600.50 & 8 & 280 & 540 \\
5 & 8638 & 2688 & 856.00 & 852.67 & 6 & 390 & 680 \\
6 & 13628 & 3960 & 833.00 & 831.12 & 9 & 363 & 781 \\
7 & 11963 & 3696 & 1185.00 & 1182.06 & 6 & 519 & 1028 \\
\hline
\end{tabular}

Table 10: Results from Model 1 with reduced number of blocks 
The best HHI values found by this procedure are the same as those obtained from Model 4 except for problem 6 for which a better solution is obtained. In addition, the best solution is generally found considerably earlier in the search.

The block modelling problem described here is essentially an optimisation problem on a graph. As such it is related to a number of other well-studied graph optimisation problems, such as maximal clique, minimum colouring or maximal independent set [?]. When $\beta=1$ a block is precisely a clique. Further, the problem addressed by Model 5 , finding the minimum number of blocks, is equivalent to finding a minimum colouring of the complement of the graph. Hence it may seem productive to explore this relationship. However objective function (19) is a coarse one which potentially admits many optimal solutions, whereas objective function (1) has more discriminatory power. Use of (19) here is simply as an aid to the primary objective of maximising HHI. Moreover methods for solving the graph optimisation problems referred to above do not appear to extend to the block modelling problem with $\beta<1$. We prefer to explore here methods which are applicable to both cases.

In a further attempt to reduce the effect of symmetry, following the disappointing performance of Models 2 and 3, the same device as in [?] was used to remove some of the arbitrariness in the labelling of the blocks. This can be done by insisting that block 1 contains one of the nodes of maximal degree, further reducing the size of the ILPs. We denote as Model 6 the model comprising Model 1 with the maximum number of blocks determined by the iterative use of Model 5 and with block 1 partially specified in this way. Table 11 shows the results from Model 6 .

\begin{tabular}{|c|r|r|r|r|r|r|}
\hline Problem & $V_{0}$ & $V_{1}$ & $\begin{array}{r}\text { Best } \\
\text { HHI }\end{array}$ & $N_{o}$ & $N_{e}$ & $\begin{array}{r}\text { Upper } \\
\text { Bound }\end{array}$ \\
\hline 1 & 182.00 & 177.90 & 78 & 47 & 7778 & 78 \\
2 & 230.00 & 226.00 & 136 & 289 & 3842 & 187 \\
3 & 319.00 & 315.28 & 125 & 75 & 1364 & 282 \\
4 & 542.00 & 539.22 & 280 & 280 & 1688 & 489 \\
5 & 782.00 & 779.33 & 390 & 37 & 1310 & 607 \\
6 & 745.50 & 751.65 & 363 & 305 & 858 & 700 \\
7 & 1099.67 & 1097.45 & 519 & 261 & 522 & 911 \\
\hline
\end{tabular}

Table 11: Results from Model 6

As Table 11 shows, the solution for Problem 1 is now a proven optimal solution (given the maximum number of blocks considered), in approximately 
88 secs. Although no better solutions are found for the remaining problems, useful reductions in both the initial and final upper bounds are obtained.

Table 12 shows the effect of combining Model 6 and Model 2, i.e. making a partial assignment to block 1 and adding constraints similar to (12) for blocks $2, \ldots, b$ and confirms the earlier finding that these constraints do not help if the search is truncated. Worse solutions pertain at termination for three of the four largest problems.

\begin{tabular}{|c|r|r|r|r|r|r|}
\hline Problem & $V_{0}$ & $V_{1}$ & $\begin{array}{r}\text { Best } \\
\text { HHI }\end{array}$ & $N_{o}$ & $N_{e}$ & $\begin{array}{r}\text { Upper } \\
\text { Bound }\end{array}$ \\
\hline 1 & 182.00 & 177.90 & 78 & 126 & 4878 & 78 \\
2 & 230.00 & 223.90 & 136 & 780 & 2295 & 172 \\
3 & 319.00 & 313.58 & 125 & 680 & 1023 & 283 \\
4 & 542.00 & 540.12 & 274 & 302 & 997 & 496 \\
5 & 782.00 & 779.41 & 390 & 444 & 690 & 600 \\
6 & 745.50 & 752.79 & 333 & 355 & 432 & 710 \\
7 & 1099.67 & 1096.67 & 507 & 299 & 434 & 932 \\
\hline
\end{tabular}

Table 12: Results from Model 6 combined with Model 2

As Table 13 shows, the combination of Model 6 with CPLEX's symmetry preprocessor allows the search to be completed for all problems except problem 6 , for which symmetry is again not detected.

\begin{tabular}{|c|c|r|r|r|}
\hline Problem & $V_{1}$ & $\begin{array}{r}\text { Best } \\
\text { HHI }\end{array}$ & $\begin{array}{r}\text { Upper } \\
\text { Bound }\end{array}$ & $\begin{array}{r}\text { Time } \\
(\text { secs })\end{array}$ \\
\hline 1 & 130.89 & 78 & 78 & 6.79 \\
2 & 167.31 & 136 & 136 & 7.16 \\
3 & 199.66 & 125 & 125 & 135.34 \\
4 & 437.62 & 280 & 280 & 396.69 \\
5 & 628.59 & 390 & 390 & 35.75 \\
6 & 751.65 & 363 & 700 & 900 \\
7 & 855.00 & 519 & 519 & 152.87 \\
\hline
\end{tabular}

\section{Table 13: Results for Model 6 with Symmetry Preprocessing}

It may be noted that running Model 6 without symmetry preprocessing finds the same HHI values, but without proving their optimality. This may give some confidence in using a solver without a symmetry preprocessing feature. 
As remarked earlier, it is possible that a better solution exists with a larger number of blocks than considered in Model 6, even if the solution found by Model 6 is 'proven optimal'. This is evidenced by comparing the result for problem 5 in Table 13 with that in Table 7 . However the approach described here offers a way of getting a good solution in reasonable time. Obtaining a proven optimal solution to instances of the block model problem is likely to be very expensive. For example, by using the approach described here, we know that the best HHI value we can obtain for problem 1 with most 6 blocks is 78. The upper bound on HHI for a 13 block solution is 76 . Hence to confirm or deny that the optimal HHI value for problem 1 is 78 would require looking at solutions with between 7 and 12 blocks. Attempts to do so using Model 6 with the addition of the constraints:

$$
\sum_{i=1}^{n} \lambda_{i k} \geq 1 \quad(k=1, \cdots, 7)
$$

were abandoned without result after 20 hours. Even proving that there is no better solution in exactly seven blocks took in excess of two hours.

\section{The Case of Non-Full Density Blocks}

When $\beta<1$ the block density constraints (3) are weaker than when $\beta=$ 1. Consequently we expect that there are likely to be many more feasible partitions of each graph and a correspondingly larger search space. Also, as noted earlier, the reductions achieved by presolve will not be as great as is the case when $\beta=1$. Additionally preliminary investigations showed that CPLEX's cuts were again ineffective and that, surprisingly, CPLEX's heuristics are not as effective in finding feasible solutions as for the full density block case. These factors may make it more difficult to find good partitions in a limited time. In recognition of this, the time limits on the branch and cut search were increased to 1200 secs for Problems 2-3 and 1800 secs for Problems 4-7.

The influence of the maximum number of blocks on the quality of solutions obtained in a limited search time can be seen by comparing Tables 3 and 10. Clearly we would expect it be possible to form fewer blocks in the non-full density case than in the full density case. If we can establish a good estimate of the smallest number of blocks for which a feasible solution exists, this would mitigate, to some extent, the potential difficulty referred to above. Although Jessop's heuristic does not directly address the case of nonfull density blocks, it can be used to derive solutions for this case. Clearly 
any feasible solution to the full block case is a feasible solution to the corresponding non-full block case and can potentially be improved as follows. Suppose $i, j$ are full density blocks with $s_{i}, s_{j}$ members respectively and that there are $e_{i j}$ edges connecting nodes in block $i$ to nodes in block $j$. The number of blocks can be reduced by 1 if:

$$
s_{i}^{2}+s_{j}^{2}+2 e_{i j} \geq \beta\left(s_{i}+s_{j}\right)^{2}
$$

as blocks $i$ and $j$ can be merged. Successive block mergers yield the results in Table 14.

\begin{tabular}{|c|c|c|}
\hline Problem & $b$ & HHI \\
\hline 1 & 5 & 110 \\
2 & 10 & 158 \\
3 & 11 & 131 \\
4 & 6 & 410 \\
5 & 4 & 626 \\
6 & 5 & 707 \\
7 & 4 & 871 \\
\hline
\end{tabular}

Table 14: Results from Heuristic for $\beta=0.8$

Unfortunately the increased size of the subproblems and the inability of the CPLEX heuristics to find feasible solutions early enough in the search causes the procedure for reducing the number of blocks to be considered, described in Section 5, to be ineffective. With the exception of Problem 1, for which a 4 block solution was found, no feasible solutions or other useful information was found within the imposed time limits of 90 secs for problems 1-3 and 120 secs for problems 4-7. We can, of course, use the number of blocks provided by the procedure for $\beta=1$, which is relatively cheap to compute, if this improves on the number of blocks found by the heuristic for any value $\beta<1$. For the problems used here this is the case for Problem 3, for which the heuristic gives 11 blocks with $\beta=0.8$ whilst the procedure of Section 5 gives a 10 block solution for $\beta=1$. Applying Model 1 with the maximum number of blocks determined in this way gives the results in Table 15 . 


\begin{tabular}{|c|c|c|c|c|c|}
\hline \multirow{2}{*}{ Problem } & \multicolumn{2}{|c|}{ After Presolve } & & Best & Upper \\
& Rows & Columns & $b$ & HHI & Bound \\
\hline 1 & 2875 & 1050 & 4 & 138 & 197 \\
2 & 13090 & 4650 & - & - & 315 \\
3 & 15883 & 5610 & 10 & 149 & 427 \\
4 & 14086 & 4920 & 5 & 542 & 719 \\
5 & 11745 & 4100 & 3 & 782 & 1002 \\
6 & 16267 & 5640 & 4 & 601 & 992 \\
7 & 13023 & 4512 & 4 & 1141 & 1396 \\
\hline
\end{tabular}

Table 15: Results from Model 1 for $\beta=0.8$

The results achieved in Table 15 suggest that satisfactory results can be obtained from Model 1 if the initial number of blocks is low. They are much worse if the number of blocks is high, as is the case for Problems 2 and 3; this is partly due to the relatively low density of the graph for these problems.

Table 16 gives the results obtained when a node of maximum degree is 'anchored' in block 1 . Unlike the case of $\beta=1$, the results with the anchored model do not weakly dominate those of the unanchored model; worse values of HHI being obtained for Problems 3 and 4 whilst a better value is obtained for Problems 5 and 7 . Each of the models fails to find a feasible solution in one case. Adding either of the symmetry breaking constraints (12) or (14) again is not beneficial to performance.

\begin{tabular}{|c|c|c|c|c|c|}
\hline & \multicolumn{2}{|c|}{ After Presolve } & & Best & Upper \\
Problem & Rows & Columns & $b$ & HHI & Bound \\
\hline 1 & 2065 & 760 & 4 & 138 & 179 \\
2 & 12219 & 4350 & 10 & 162 & 278 \\
3 & 14922 & 5280 & 10 & 141 & 401 \\
4 & 13383 & 4680 & 5 & 540 & 658 \\
5 & 8943 & 3120 & 3 & 806 & 909 \\
6 & 15576 & 5405 & - & - & 936 \\
7 & 12470 & 4324 & 4 & 1141 & 1305 \\
\hline
\end{tabular}

Table 16: Results from anchored Model 1 for $\beta=0.8$

CPLEX's symmetry preprocessing does not help as much as it did in the full density case. Table 17 shows that, although a proven optimal solution was obtained (in 577 secs) to problem 1, no IFS was found before termination 
in three of the other cases and, in the remaining three, worse solutions were obtained.

\begin{tabular}{|c|r||r|}
\hline Problem & $\begin{array}{r}\text { Best } \\
\text { HHI }\end{array}$ & $\begin{array}{r}\text { Upper } \\
\text { Bound }\end{array}$ \\
\hline 1 & 138 & 138 \\
2 & - & 273 \\
3 & - & 162 \\
4 & 450 & 658 \\
5 & 782 & 916 \\
6 & - & 936 \\
7 & 1069 & 1278 \\
\hline
\end{tabular}

Table 17: Results for anchored Model 1 for $\beta=0.8$ with Symmetry Preprocessing

Although better HHI values are obtained via ILP for those problems for which feasible solutions are found, the failure of the ILP approaches to always find feasible solutions is problematic.

\section{Conclusions}

The results given in Table 11 suggest that, despite the weakness of the LP relaxation, ILP does provide a means of getting substantially better quality solutions to full-density problems of up to 50 nodes than does Jessop's heuristic in a reasonable amount of time. Larger problems may well be problematic unless some problem specific cuts can be developed to tighten the LP relaxation. This is even more important for the non-full density block problem as the size of the resulting ILP may make it difficult to find good solutions, even if only a small number of blocks need be formed. Finding good solutions to the non-full density remains challenging.

The results reported here also suggest that we still do not understand how to effectively handle symmetry in ILP. Some doubt is cast on the general effectiveness of the symmetry breaking inequalities suggested by Sherali and Smith [?] in cases in which it is not viable to allow a complete branch and cut search. Margot's work [?] on embedding symmetry breaking features within a branch-and cut algorithm, which to some extent parallels developments in constraint programming [?], may provide a more satisfactory approach. 


\section{Acknowledgements}

I am grateful to Alan Jessop for helpful discussions, provision of data and for supplying me with the code for his heuristic. I am also grateful to the anonymous referees whose comments and suggestions were most helpful.

\section{References}

[1] NEOS Optimization Software Guide. http://wwwfp.mcs.anl.gov/otc/Guide/SoftwareGuide/, November 2004.

[2] I P Gent and B M Smith. Symmetry breaking during search in constraint programming. In W Horn, editor, Proceedings of ECAI-2000, pages 599603. IOS Press, 2000.

[3] A Jessop. Multiple attribute probabilistic assessment of the performance of some airlines. In M Koksalan and S Zionts, editors, Multiple Criteria Decision Making in the New Millenium, pages 417-426. Springer, Berlin, 2001.

[4] A Jessop. Exploring structure: a blockmodel approach. Civil Engineering and Environmental Systems, 19:263-284, 2002.

[5] A Jessop. Blockmodels with maximum concentration. European Journal of Operational Research, 148:56-64, 2003.

[6] K E Petrie, B M Smith, and N Yorke-Smith. Dynamic symmetry breaking in constraint programming and linear programming hybrids. Technical Report APES-81-2004, Apes Research Group, University of St Andrews, 2004.

[7] L G Proll. Formulation of integer linear programs - an example. International Journal of Mathematical Education in Science and Technology, 20:415-420, 1989.

[8] L G Proll and B M Smith. ILP and constraint programming approaches to a template design problem. INFORMS Journal on Computing, 10:265-275, 1998.

[9] C T Ragsdale and G W Shapiro. Incumbent solutions in branch-andbound algorithms: setting the record straight. Computers and Operations Research, 23:419-424, 1996. 
[10] H D Sherali and J C Smith. Improving discrete model representations via symmetry considerations. Management Science, 47:1396-1407, 2001.

[11] H P Williams. Model Building in Mathematical Programming. John Wiley and Sons, 4th edition, 1999. 